\title{
DYNAMICS OF ELASTIC HALF-PLANE WHEN RESETTING THE VERTICAL STRESS AT THE CRACK
}

\author{
Lyudmila A. Alexeyeva ${ }^{1}$, Bakytbek T. Sarsenov ${ }^{2} \S$ \\ ${ }^{1}$ 050010, Kazakhstan, Almaty, Pushkin Str. 125 \\ ${ }^{2} 161200$, Kazakhstan, Turkistan, Sattarkhanov str. 29
}

\begin{abstract}
We consider a model problem for the research of Earth's surface state caused by discharge of tectonic stress on the deep cracks in the earth's crust. Using the numerical method of bicharacteristics, algorithm is developed and solution of the problem of non-stationary dynamics of a homogeneous isotropic elastic half-space is built under plane strain when one resets the vertical stress on crack. Diffraction of waves on the surface is studied, pictures of wave fields and stress - strain state of the medium and the surface for different moments of time are built.
\end{abstract}

AMS Subject Classification: 65M06, 65M25

Key Words: method of bicharacteristics, problem of non-stationary dynamics, homogeneous elastic half-space, diffraction of waves, plane strain

\section{Introduction}

Mathematical modeling of processes of propagation and diffraction of waves in different bodies and media refers to the actual problems of mathematical physics. Processes of propagation of harmonic waves and stationary diffraction of waves in media with the canonical boundary surfaces are the most studied. Non-stationary wave processes in deformable solid media are much less studied. To solve the non-stationary problems in elastic media various numeri-

Received: August 19, 2015

Published: April 19, 2016

${ }^{\S}$ Correspondence author (c) 2016 Academic Publications, Ltd.

url: www.acadpubl.eu 
cal and numerically-analytical methods: spatial characteristics, finite elements, boundary integral equations, and others are widely used. Difference method, using the method of spatial characteristics, previously has been proposed by R.Clifton in [1] to study of planar dynamical problems, and in [2] it has been developed by W.Recker to study the propagation of elastic waves in isotropic bodies of rectangular shape. One of the most user-friendly methods in applications is the bicharacteristics method, using the ideas of the splitting method, developed by G.Tarabrin [3]. In this paper, based on this method, a solution of the problem of non-stationary dynamics of a homogeneous isotropic elastic half-space is constructed with a crack in the plane strain. This problem is the model to study of Earth's surface state due to discharge of tectonic stress on the deep cracks in the earth's crust during earthquakes.

\section{Statement of the Problem. Equations of Motion}

Let elastic isotropic medium $D$ with coefficients of Lame $\lambda, \mu$ and density $\rho$ take half-space $x_{1} \geq 0$. Consider the dynamics of the medium in a plane strain when voltage reset on a horizontal crack $S$, which is located at depth $L\left(x_{1}=\right.$ $\left.L,\left|x_{2}\right| \leq d\right)$. Assume that in initial moment the medium is at rest:

$$
\mathbf{u}=0, \dot{\mathbf{u}}=0 \quad \text { when } \quad x_{2} \geq 0,
$$

and the boundary of the half-space (full surface) is free from external loads:

$$
\sigma_{11}=\sigma_{12}=0 \quad \text { when } \quad x_{1}=0 .
$$

Hereinafter, $\mathbf{u}=\left(u_{1}, u_{2}\right)-$ move of the medium, $\sigma_{i j}-$ stress tensor, $x_{i}-$ Cartesian coordinates of the points of the medium $\mathbf{x}=\left(x_{1}, x_{2}\right), t$ - time.

By indexes after the comma we denote the partial derivatives with respect to Cartesian coordinates, and points from the top are the partial derivatives with respect to time. For convenience, we will use the Latin and Greek indices, that take the values 1,2. By the repeated Greek indices in the product the summation (convolution of the tensor) holds, but by the repeated Latin indices the summation does not hold.

Since there are no sources of fluctuations at infinity, then the requirement that at infinity the following conditions of attenuation hold:

$$
u_{j} \rightarrow 0, \sigma_{i j} \rightarrow 0 \quad \text { when } \quad\|x\| \rightarrow \infty .
$$

Moving of the medium satisfies the equations of the motion [4]:

$$
\sigma_{i \beta, \beta}+F_{i}=\rho \dot{v}_{i}
$$


where $\dot{v}_{i}(i=1,2)$ are the component vectors of the velocity $\mathbf{v} ; F_{i}(i=1,2)$ are projections of mass forces on the corresponding axes.

Connection between the components of stress tensor and the components of moving vector is expressed by Hooke's law [4]:

$$
\sigma_{i j}=\lambda u_{\beta, \beta} \delta_{i j}+\mu\left(u_{i, j}+u_{j, i}\right)
$$

where $\delta_{i j}(i, j=1,2)$ is the Kronecker delta.

It is required to determine stress-strain state of the medium and the surface at the strain relief on crack.

The center of seismic disturbances is specified by components of mass force $\mathbf{F}$, which are determined by a singular generalized function, that is a simple layer on the crack $S[5]$, where they have the following form:

$$
F_{i}=n_{\beta}\left[\sigma_{i \beta}\right]_{S} \delta_{S}(x)=n_{\beta}\left[\sigma_{i \beta}\right]_{S} \delta\left(x_{1}-L\right) H\left(d-\left|x_{2}\right|\right)
$$

where the expression in brackets is jump of components of the stress tensor on the crack, $\mathbf{n}$ is a unit normal to the surface, in this case $\mathbf{n}=\left(n_{1}, n_{2}\right)=$ $(1,0), H\left(x_{1}\right)$ is the Heaviside function, $\delta\left(x_{1}\right)$ is the Dirac delta function. We assume that the jump of stress on the crack is known:

$$
n_{\beta}\left[\sigma_{i \beta}(x)\right]_{S}=P_{i}(x, t), \quad x \in S, t>0 .
$$

There we take into account that

$$
P_{i}(x, t)=P t \exp (-a t) H(t)
$$

where $a>0$.

Here, in the calculations for submission of the $\delta$ - Dirac function, we use the delta-sequence $\delta_{\varepsilon}(x)[6]$ :

$$
\lim _{\varepsilon \rightarrow 0} \delta_{\varepsilon}(x)=\delta(x), \quad \delta_{\varepsilon}(x)=\left\{\begin{array}{l}
(2 \varepsilon)^{-1}, \quad x \in[-\varepsilon, \varepsilon] \\
0, \quad x \notin[-\varepsilon, \varepsilon]
\end{array}\right.
$$

where $\varepsilon>0$ i.e. in the calculations

$$
F_{i}=F_{i}^{\varepsilon}=n_{\beta}\left[\sigma_{i \beta}\right]_{S} \delta_{\varepsilon}\left(x_{1}-L\right) H\left(d-\left|x_{2}\right|\right)
$$




\section{Determining System of Equations}

Equation (1), which describes the small dynamic deformations in plane strain, with the Hooke's law (2), can be represented in the form of a hyperbolic system of linear differential equations in partial derivatives of the first order concerning to the velocity component vector and the stress tensor [7]:

$$
\left\{\begin{array}{l}
\dot{\bar{v}}_{i}=\bar{\sigma}_{i \beta, \beta}+\bar{F}_{i}, \\
\dot{\bar{\sigma}}_{i j}=\bar{\gamma}_{i j} \bar{v}_{i, j}+\bar{\gamma}_{33}\left(\bar{v}_{\beta, \beta}-\bar{v}_{i, j}\right) \delta_{i j}+\bar{\gamma}_{j i} \bar{v}_{j, i}\left(1-\delta_{i j}\right),
\end{array}\right.
$$

Here, for convenience of the calculations we introduce the following independent dimensionless variables and unknown quantities:

$$
\begin{gathered}
\bar{t}=\frac{t c_{1}}{L}, \bar{x}_{i}=\frac{x_{i}}{L}, \bar{v}_{i}=\frac{1}{c_{1}} \frac{\partial u_{i}}{\partial t}, \bar{\sigma}_{i j}=\frac{\sigma_{i j}}{\rho c_{1}^{2}}, \bar{F}_{i}=\frac{F_{i} L}{\rho c_{1}^{2}} ; \\
\bar{\gamma}_{12}=\bar{\gamma}_{21}=\left(\frac{c_{2}}{c_{1}}\right)^{2}, \bar{\gamma}_{11}=\bar{\gamma}_{22}=\left(\frac{c_{1}}{c_{1}}\right)^{2}=1, \bar{\gamma}_{33}=1-2 \bar{\gamma}_{12},
\end{gathered}
$$

where $c_{1}$ and $c_{2}$ are speeds of propagation of the longitudinal and transverse waves in a continuous medium, $L$ is the characteristic length. Furthermore, the bar over dimensionless parameters will be omitted.

\section{Bicharacteristics and Conditions over Them. Resolution System of Equations}

To obtain equations of the bicharacteristics and conditions over them, we split the two-dimensional system (6) on the one-dimensional. It is possible, if in the system we alternately fix one of the spatial variables. This method is consistent with the idea of K.A. Bagrinovskii and S.K. Godunov about split of multi-dimensional hyperbolic system on one-dimensional systems [7].

When $x_{\alpha}=\operatorname{const}(\alpha=1,2 ; j \neq \alpha)$, we have:

$$
\left\{\begin{array}{l}
\dot{v}_{i}-\sigma_{i j, j}=a_{i j}, \\
\dot{\sigma}_{i j}-\lambda_{i j}^{2} v_{i, j}=b_{i j},
\end{array} \quad(i=1,2),\right.
$$

where $a_{i j}=\sigma_{i \beta, \beta}-\sigma_{i j, j}+F_{i}, \quad \lambda_{i j}=\sqrt{\gamma_{i j}}, \quad b_{i j}=\gamma_{33}\left(v_{\beta, \beta}-v_{i, j}\right) \delta_{i j}+\gamma_{j i} v_{j, i}(1-$ $\left.\delta_{i j}\right)$.

Using the expression of the total differential, we exclude the partial derivatives with respect to $t$, and making some algebraic transformations, system of 
equations (7) can be transformed to the system of equations of the following form:

$$
\left\{\begin{array}{l}
-\frac{d x_{j}}{d t} v_{i, j}-\sigma_{i j, j}=a_{i j}-\frac{d v_{i}}{d t} \\
-\lambda_{i j}^{2} v_{i, j}-\frac{d x_{j}}{d t} \sigma_{i j, j}=b_{i j}-\frac{d \sigma_{i j}}{d t},
\end{array} \quad(i, j=1,2) .\right.
$$

Slope of the bicharacteristics is defined by the condition:

$$
\left|\begin{array}{cc}
-\frac{d x_{j}}{d t} & -1 \\
-\lambda_{i j}^{2} & -\frac{d x_{j}}{d t}
\end{array}\right|=0 \quad(i, j=1,2)
$$

and differential relations to the characteristics follows from the conditions:

$$
\left|\begin{array}{cc}
a_{i j}-\frac{d v_{i}}{d t} & -1 \\
b_{i j}-\frac{d \sigma_{i j}}{d t} & -\frac{d x_{j}}{d t}
\end{array}\right|=0 \quad(i, j=1,2) .
$$

Procedures, to obtain difference system of equations for (6) with respect to unknowns $\sigma_{i j}$ and $v_{i}(i, j=1,2)$ at the node points $A$ of the test body at the time $t_{n}+\tau$, differ for internal and boundary points of the studied domain (see details in [7]).

The developed method for solving dynamic problems allows to determine the velocity $v_{i}$ and the components of stress tensor $\sigma_{i j}$ at the point $A$ on any layer of time $t=t_{0}+\tau$, if we know their values on the previous layer $t=t_{0}$.

To simulate the radiation conditions at infinity we arbitrarily set boundaries, that are far enough away from the cracks, and in the target time interval $(0, T)$ the shock waves cannot reach up to them. We set three boundaries, the first two are set by the equation $\left|x_{2}\right|=6 L+2 d$ (from the right and left sides of the body), and the third one is given by the equation $x_{1}=7 L$ (the lower boundary), and we put them the following conditions:

$$
\sigma_{\alpha j}=0, \quad v_{\alpha}=0 \quad(\alpha, j=1,2)
$$

\section{Dynamics of the Medium when One Resets the Vertical Stresses on Crack}

The calculation was performed with the dimensionless parameters: $\rho=1, c_{1}=$ $\sqrt{\gamma_{11}}=1, c_{2}=\sqrt{\gamma_{12}}=0.577$ (relationship of parameters: $c_{2}: c_{1}=1: \sqrt{3} \approx$ 0.577 , i.e. $\lambda=\mu$ is used for soil in the seismology [4]), with the depth and 


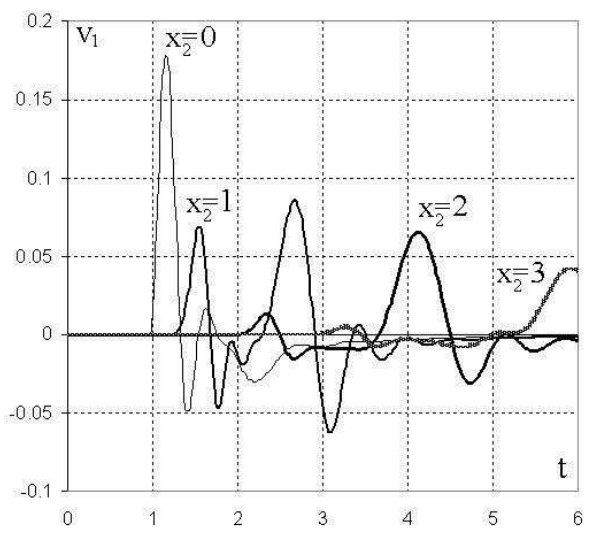

a

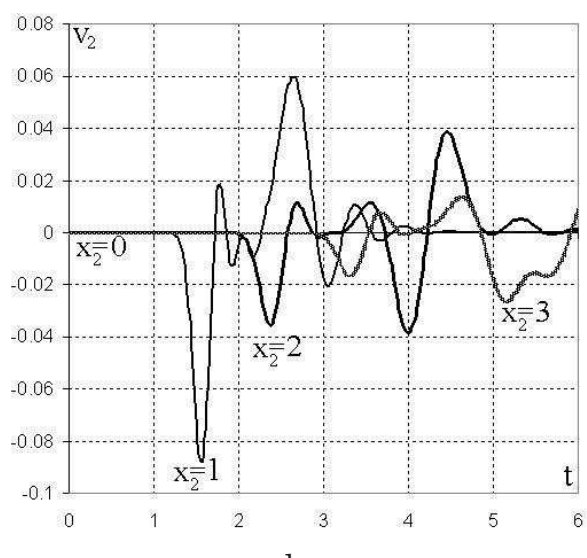

$\mathrm{b}$

Figure 1: Oscillograms of the velocities $v_{1}(\mathrm{a})$ and $v_{2}(\mathrm{~b})$ on the surface, when $x_{1}=0, x_{2}=0 ; 1 ; 2 ; 3$

width of cracks $L=1, d=0.1$, respectively, at the time interval $(0,6]$. Power surge in the crack is given as follows

$$
P_{1}(x, t)=20 \cdot t \cdot \exp (-10 t) \cdot H(t), P_{2}(x, t)=0 .
$$

In the calculations $\varepsilon=h$, and steps on the space-time grid: $h_{1}=h_{2}=h=$ $0.05, \tau=0.025$.

The velocities of longitudinal and transverse waves in dimensionless values are $c_{1}=1$ and $c_{2}=0.577$, respectively. Therefore, the body (longitudinal) and shear (transverse) waves from the crack to the point $O(0,0)$ will come at $t_{1}=1$ and $t_{2}=1.73$, respectively, and to the point $A(0,1)$ at $t_{1}=1.35$ and $t_{2}=2.33$, to the point $B(0,2)$ at $t_{1}=2.15$ and $t_{2}=3.71$, and to the point $C(0,3)$ at $t_{1}=3.07$ and $t_{2}=5.31$. In Figure 1 at the indicated moments of time we can see the beginning of the burst rate of fluctuations. At the epicenter (the point O) $v_{2}=0$, since discharge voltage occurs symmetrically and parallel to the axis $O x_{1}$.

Figure 2 shows oscillograms of the stress $\sigma_{22}$ on the surface $x_{1}=0(a)$ and on the depth $x_{1}=0.5(b)$ at points $x_{2}=0, x_{2}=1, x_{2}=2, x_{2}=3$. Stresses change sign: in the epicenter it is mostly tensile stresses. However, far from it on the surface oscillation process occurs with the change of compressive stresses in the tensile and vice versa.

In Figure 3, we show oscillograms of the velocities $v_{1}$ and $v_{2}$ on the depth $x_{1}=0.5$ at points $x_{2}=0, x_{2}=1, x_{2}=2, x_{2}=3$. Comparing them with the 


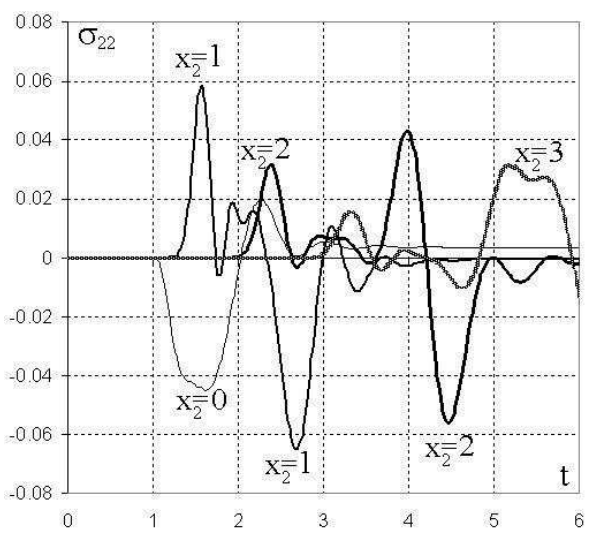

a

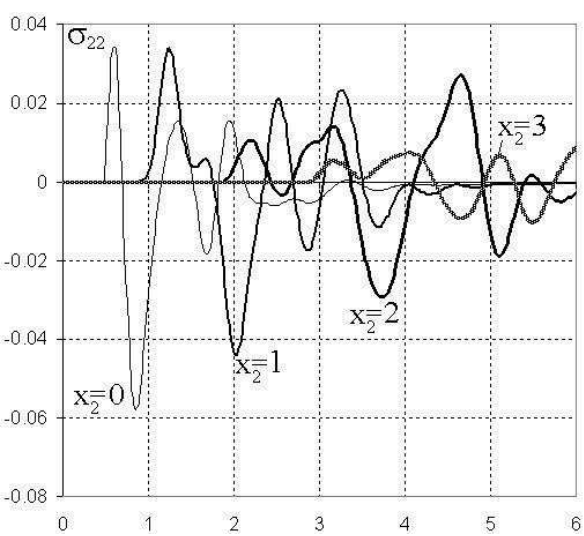

b

Figure 2: Oscillograms of the stress $\sigma_{22}$ on the surface $x_{1}=0(a)$ and depth $x_{1}=0.5(b)$, when $x_{2}=0 ; 1 ; 2 ; 3$

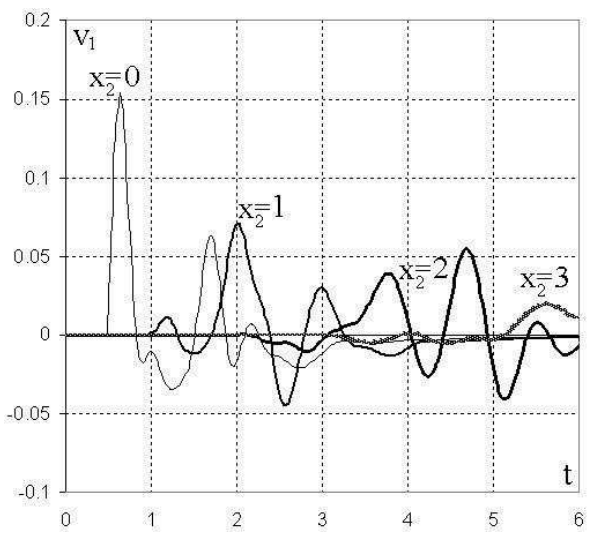

a

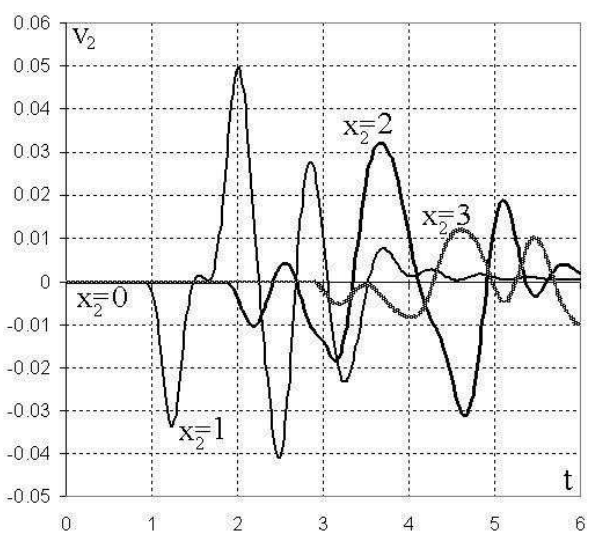

b

Figure 3: Oscillograms of the velocities $v_{1}(a)$ and $v_{2}(b)$ on the depth $x_{1}=0.5\left(x_{2}=0 ; 1 ; 2 ; 3\right)$

oscillograms on the surface (Figure 1), we can see similarity of the graphs. On the surface the vibration amplitude is more than on the depth.

In the Figure $4-5$, we show sections of the velocity $v_{1}$ and stresses $\sigma_{11}$ and $\sigma_{22}$ on the plane $x_{2}=0$ (due to symmetric property of effect on the plane: $\left.v_{2}=0\right)$ at the moments $t=1, t=1.5$ and $t=2$.

It can be noted, that at the moment $t=1$ the wave only reaches up to 

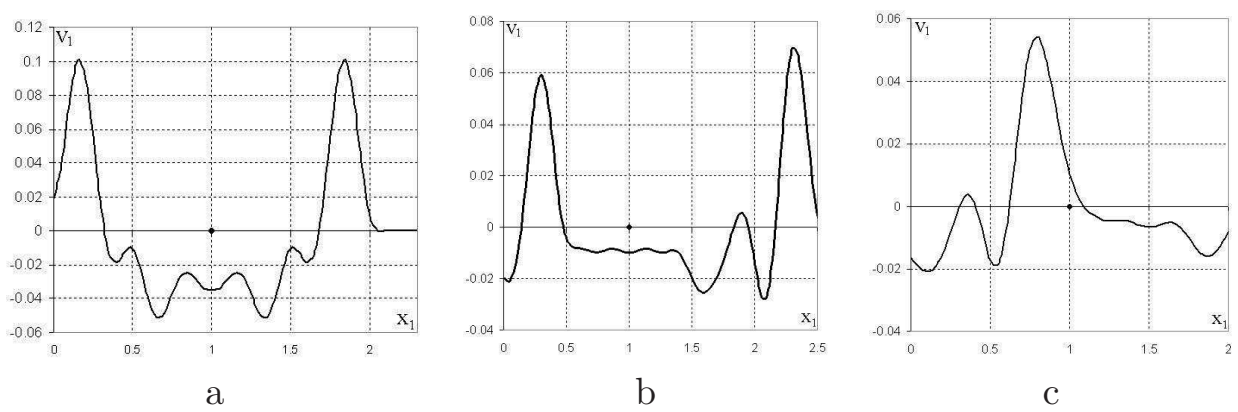

Figure 4: Section of the velocity $v_{1}$ on the plane $x_{2}=0$, at the moments $t=1(a), t=1.5(b), t=2(c)$

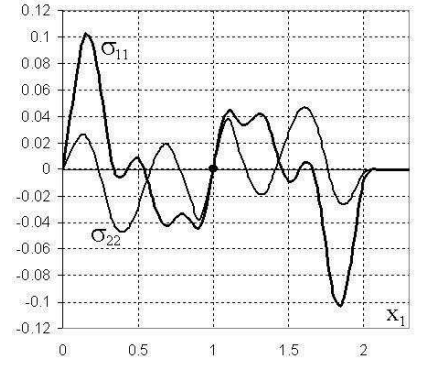

a

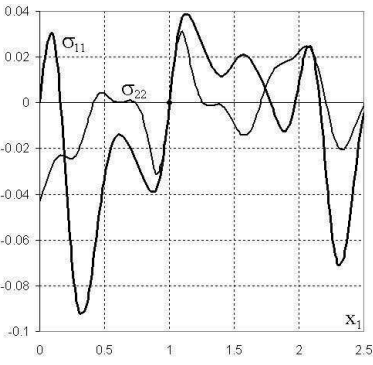

$\mathrm{b}$

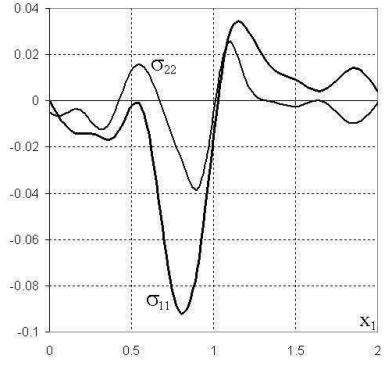

$\mathrm{C}$

Figure 5: Sections of the stresses $\sigma_{11}$ and $\sigma_{22}$ on the plane $x_{2}=0$, at the moments of time $t=1(a), t=1.5(b), t=2(c)$

the ground surface (there is no reflected wave) and the velocity $v_{1}$ is, still, symmetric, and stresses $\sigma_{11}$ and $\sigma 22$ are antisymmetric with respect to an axis, extending along the crack, which corresponds to the present action. Moreover, this moment corresponds to the moment, when external influence is removed, and the medium begins to freely oppose.

At time $t=1.5$ the wave, reflected from the surface, reaches the middle of the distance to the crack, and at time $t=2$ it reaches the crack, and the symmetry of the graph is lost. Comparing the figures of amplitude fluctuations, we can see that, in process of time the amplitude decreases, due to the fact that in these moments there is no external influence, and the medium opposes, causing a wave to fade (the wave loses its energy).

Figure 6 shows the vector field of velocities in medium when one resets the vertical stresses on the crack. At the initial time two shock waves arise on the crack: the body wave that moves with velocity $c_{1}$, and the shear wave with 


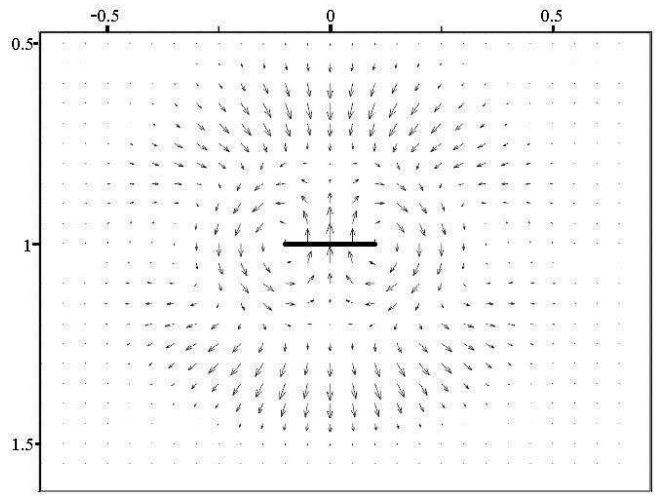

a

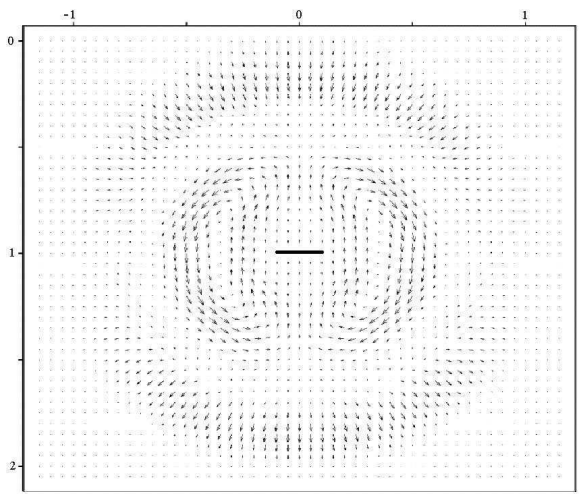

b

Figure 6: Vector field of velocities before the reflection of shock waves; $t=0.5(a) ; t=1(b)$

the velocity $c_{2}$. The body waves are ahead of shear waves. In the figures, the annular zones in the neighbourhood of the crack correspond to them. At the front of the shock waves the conditions for the races hold [8]:

$$
\left[v_{j}\right]_{F r_{k}}=-\left(\rho c_{k}\right)^{-1}\left[\sigma_{i j}\right] n_{i} \quad(k=1,2) .
$$

The waves are weak shock, since the voltage reset is continuous and starts from scratch. In front of the first shock wave before it reaches the surface the medium is at rest.

At $t=0.5$ the body wave runs the distance 0.5 , and the shear wave is 0.29 as we can see in Figure 6a. It can be seen that at the ends of the crack the vortex field is formed, that characterizes the shear waves, and the shear wave is not spread from the middle part of the crack by the perpendicular direction, and the body wave is not spread from the ends of the crack in directions which are parallel to the crack. This is due to the type of impact on the crack. The edges of the crack act as sources of cylindrical shear waves.

In Figure 6, the waves have not yet reached the ground surface. In Figure $6 \mathrm{~b}$, it can be seen that the wave ran up to the surface and has not been reflected yet (it is preserved complete symmetry with respect to the crack).

Figure $7 \mathrm{a}$ shows the moment when the wave must be reflected from the free boundary and reach the middle of the segment, connecting with a crack, and this process is observed in the figure. In Figure $7 \mathrm{~b}$, we can see the moment when the reflected body wave ran up the crack, and the shear wave, passing through the reflected wave (with some distortions), ran up to the surface and 


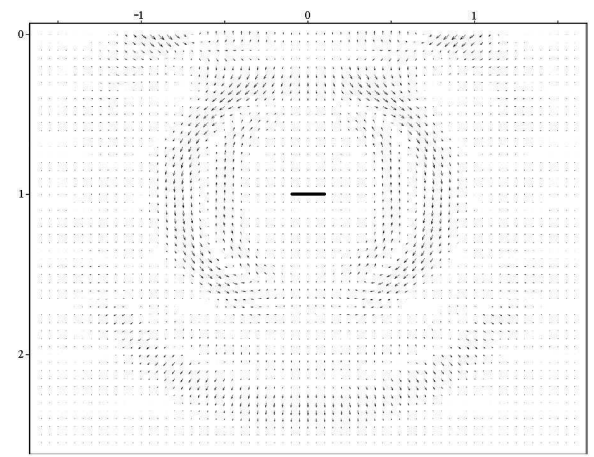

a

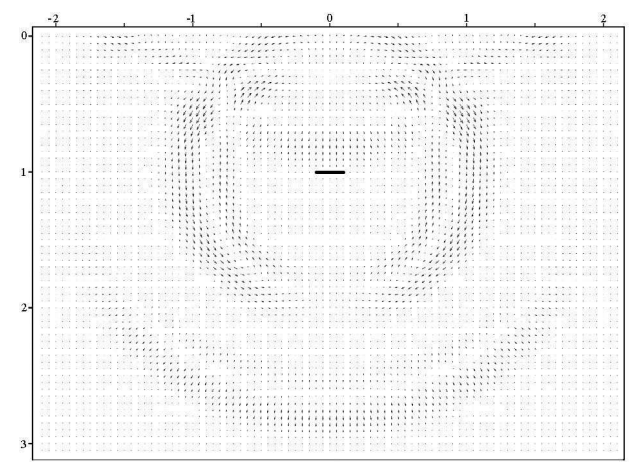

$\mathrm{b}$

Figure 7: Vector field of velocities after the reflection of shock waves: $t=1.5(a) ; t=2(b)$

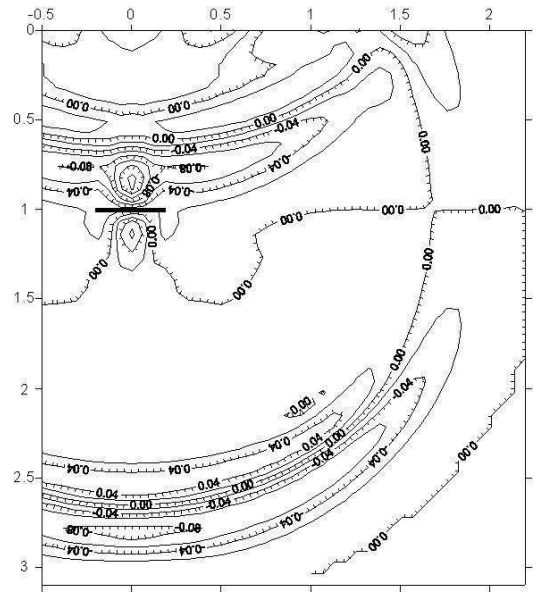

a

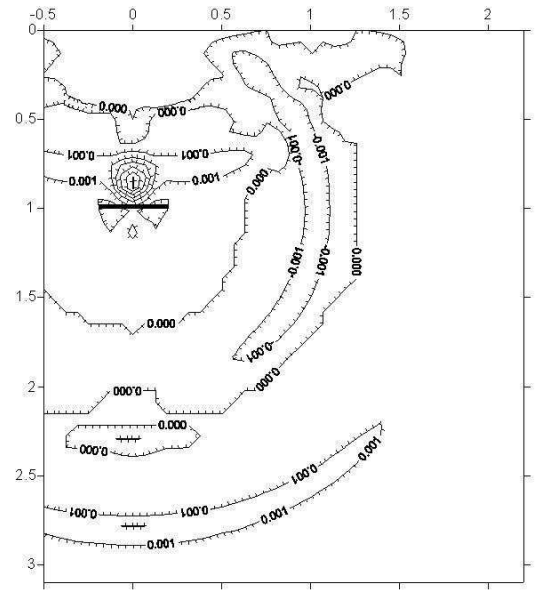

$\mathrm{b}$

Figure 8: Isolines of the first (a) and second (b) stress invariants: $t=2$

began a process of reflection. A complex diffraction pattern of interaction of the falling and reflected shock waves arises, a complex stress-strain state of the medium, which is shown in Figure 8, is formed.

Interaction of body and shear waves with the surface and between themselves (after reflection) generate different waves, including Rayleigh waves. In Figure 7 , we can see how the wave starts to form along the surface, forming a Rayleigh surface wave. In this media, it spreads with the speed of $c_{R}=0.918 c_{2}$, i.e., close to the velocity of shear wave propagation. Waves coming from the 


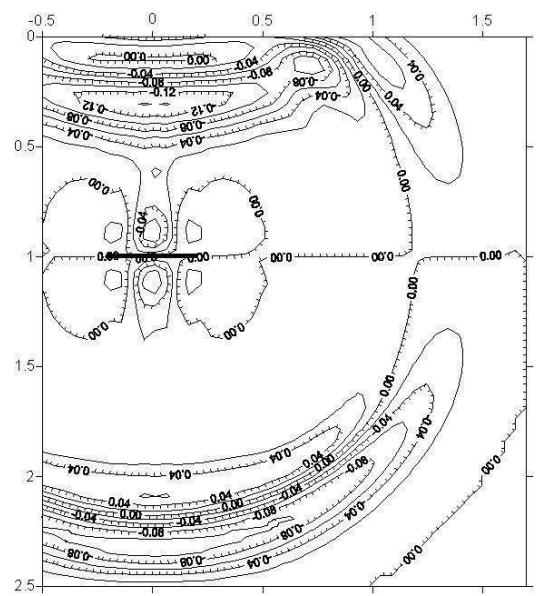

a

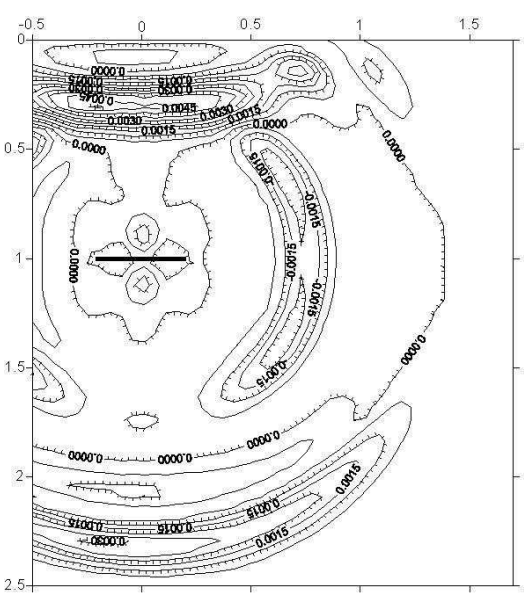

$\mathrm{b}$

Figure 9: Isolines of the first (a) and the second (b) stress invariants $t=1,5$

crack-down without encountering obstacles, are scattered with depth and over time.

In Figure 9 and 8, isolines of the first (a) and second (b) invariants of the stress tensor at the moments of time $t=1.5$ and $t=2$ are constructed. The first invariant determines the pressure in the medium, the second one - the intensity of the shear stresses. As we can see, the voltage in the upper zone from the diffraction of waves on the surface in one and a half times higher than the voltage in the free lower zone of the dispersion of elastic waves (0.12 and 0.8 for (a) and 0.0045 and 0.0030 for (b)).

\section{References}

[1] R.J. Clifton, A difference method for plane problems in dynamic elasticity, Quarterly of Appleid Mathematics, 25 (1967), 97-116.

[2] W.W. Reker, A numerical solution of three-dimensional problems in dynamic elasticity, Journal of Applied Mechanics, 37, No. 1 (1970), 116-122.

[3] G.T. Tarabrin, Application of bicharacteristics method to solve non-stationary problems in dynamics of anisotropic arrays, Structural Mechanics and Calculation of Structures, 4 (1981), 38-43.

[4] W.K. Nowacki, Theory of Elasticity, Mir, Moscow, (1975).

[5] L.A. Alexeeva and I.Sh. Dildabayeva, Generalized solution of dynamic equations of the elastic medium with curvilinear crack during the plane deformation, Mathematical Journal, 7, No. 2(25) (2007), 19-31. 
[6] V. Kech and P. Teodorski, Introduction to Theory of Generalized Functions with Applications in Techniques, Mir, Moscow (1978).

[7] S.S. Juzbayev and B.T. Sarsenov, Mathematical Journal, 3, No. 1(7) (2003), 55-62.

[8] G.I. Petrashen, Wave propagation in anisotropic elastic media, Nauka, Moscow (1980). 